\title{
Design and analysis of novel cutter roller of branches chopping machine
}

\author{
Zhu Zhifang ${ }^{12}$ \\ 1.Jiangxi Province Key Laboratory of precision drive and control,Nanchang,330099, China \\ 2.Nanchang Institute of Technology,Nanchang,330099,China
}

Keywords: branch chopper; knife roller; involute

Abstract.: Traditional branches chopped finely chopped device volume is larger, cutting tool wears, big impact. This article is based on the shortcomings of traditional cutter, and put forward a kind of reduced chopped device volume, reduce the impact and abrasion of involute arc type knife plate. After the arc can make the involute type knife cutting branches arc in contact with the knife, not reduce impact and abrasion. The knife roller device by six involute knife stick a patchwork, reduce the blade by stress, prolong service life.

\section{Introduction}

With the development of industry and the oil resources crisis arising, the demand of new power generation is more urgent, and green energy development is the strategy of our country. Biomass energy is one kind of green renewable energy, so it is a very good choice for the development and utilization of biomass energy. Biomass energy is extensive and exists in all kinds of animals and plants, like the abandoned branches among which have large quantity of biomass energy. Before turning branches into available energy, it needs special processing equipment to chop the branches into small pieces. Here introduce a new branch chopping machine which is biomass power generation preprocessing equipment. The design of the branches chopping mechanism as the main part of this machine is much related to the quality of the whole machine. Most of the traditional chopping mechanism is cutter disc structure and circular cutter role structure. The cutter disc structure chopping mechanism has a huge body that make the whole machine very heavy. Although the circular cutter role structure chopping mechanism has a small bulky, the role is easy to burst and make large noise. So the development of a new type of chopping mechanism that can solve these two problems is of great significance. This paper introduce a new type of involute cutter roller design, which used involute broadsword and can not only reduce the impact happened inside the machine, but also can reduce machine vibration and improve efficiency of chopping ${ }^{[1] .}$

\section{Principle of new branches chopped cutter roller}

According to the survey in the southern mountainous areas, it shows that the south is hilly and mountainous areas, suitable for small branches chopped machine operation, therefore the cutter disc structure is not appropriate, and the circular cutter roller structure is easy to have a big impact. In order to use cutter roller structure as new branches chopper mechanism, the circular cutter roller chopping mechanism need an innovative design. Through the analysis of the structure of circular cutter, it finds that the impact happens while the cutter roller branches chopped branches, because the branches contact with the cutter roller during processing ${ }^{[2]}$.

Through the analysis of the shortcomings of circular cutter, to solve the impact problem, it has to make the branches not contact with the cutter roller after chopping. As the branches are doing linear motion, it requires the arc curve edge curvature of the cutter roller is constantly changing to avoid the branches contact with the cutter. So an involute curve as a curvature changing constantly curve is applied in this paper ${ }^{[3] .}$ Involute form as shown in Figure 1, from the diagram can be seen 
that the curvature of each point on the involute is different.

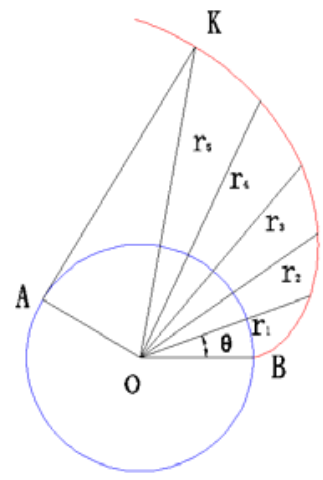

Fig.1 variation of Involute curvature

\section{Design and analysis of cutter roller structure of branches chopping machine}

\section{A Design of cutter roller structure of branches chopping machine}

The end view drawing of the cutter disc of the branches chopping machine is illustrated in Fig.2. As can be seen from Fig.2, the cutter disc includes base circle, blade, compensation arc, compensation curve, involute blade arc and shaft hole. The working principle of the cutter disc is that the blade chops the branches by the inertial force while the cutter roller rotates. The broken branches and branches relative to the cutter involute arc, because of its curvature along with the rotation has been in reducing, so branches and the cutter shift and the impact decreases. Because the involute curve and base circle may not be tangent, so the cutter head is designed with compensation and compensation arc curve.

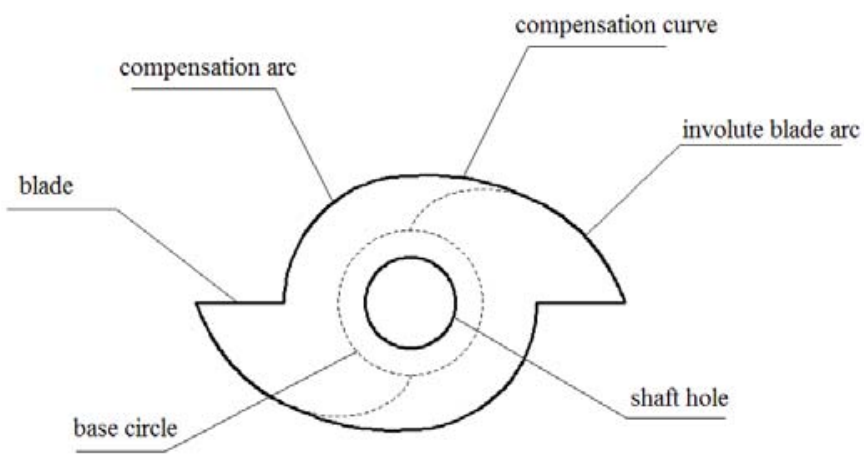

Fig. 2 end view drawing of cutter disk

The cutter shift assembles of branches chopping machine is composed of six of the involute cutter disc, and the angle between each cutter wheel is 30 degrees of dislocation. Such structure effective scattered the work stress of each cutter, so as to prolong the life of cutter. The structure diagram is shown in Fig.3. 


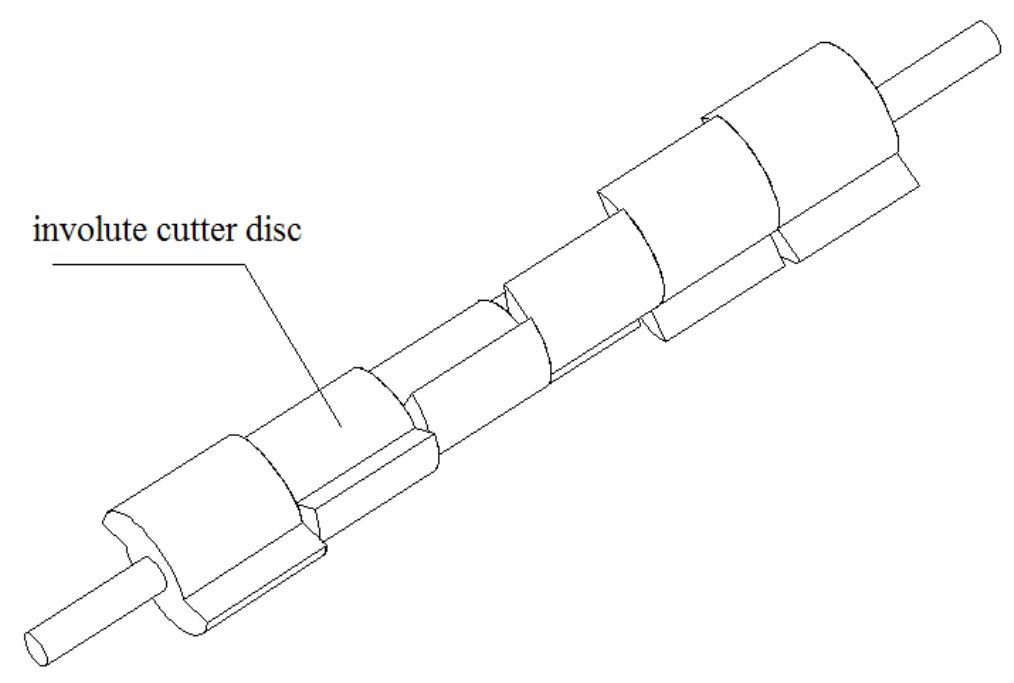

Fig. 3 the structure of cutter shift

\section{$B$ analysis of cutter roller structure of branches chopping machine}

The mathematical analysis of the cutter chopping the branches is illustrated in Fig. $4^{[4,5] \text {, }}$ where the radius of the base circle is $r$, involute curvature of A point in the blade is $R_{\mathrm{A}}$, the pressure angle is $\alpha_{\mathrm{A}}$, unfold angle $\theta=90^{\circ}$, the angular velocity is $\omega$, material speed is $\mathrm{V}$, and the involute curvature is $\mathrm{R}$.

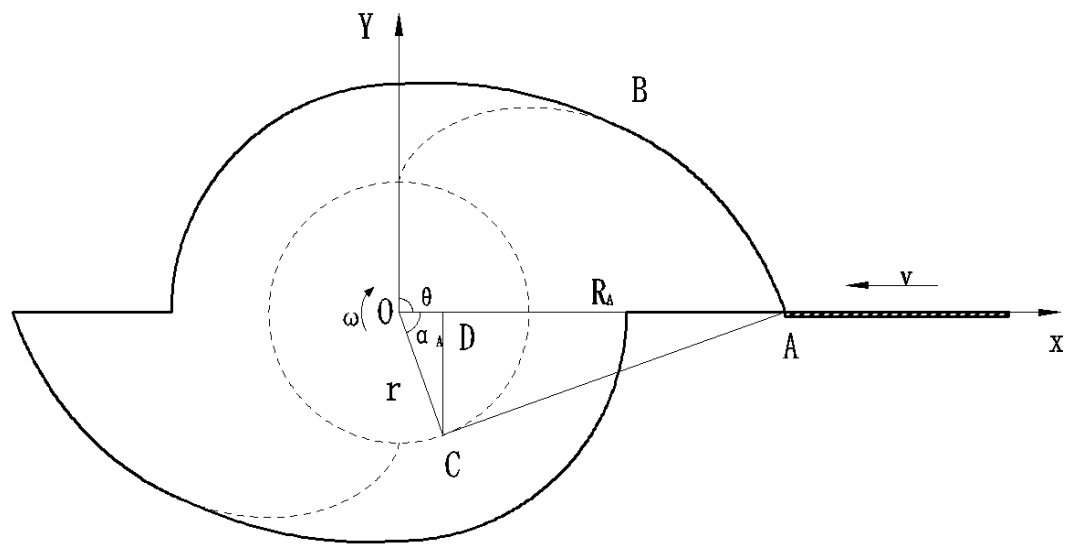

Fig.4 cutting coordinate diagram

The involute length of the involute is:

$$
\mathrm{S}=\pi \mathrm{r}\left(\frac{\theta-\omega \mathrm{t}}{180}\right)
$$

The parameter equation of involute is:

$$
\left\{\begin{array}{l}
X_{1}=\mathrm{r} \cos (\alpha-\omega \mathrm{t})+\mathrm{S} \sin (\alpha-\omega \mathrm{t}) \\
Y_{1}=\mathrm{rsin}(\alpha-\omega \mathrm{t})-\mathrm{S} \cos (\alpha-\omega \mathrm{t})
\end{array}\right.
$$

The curvature of the involute is:

$$
R=\sqrt{X_{1}^{2}+Y_{1}^{2}}
$$

Simultaneous (1) (2) (3):

$$
R=\sqrt{r^{2}+S^{2}}=\sqrt{r^{2}+\left[\frac{\pi r(\theta-\omega t)}{180}\right]^{2}}
$$


To make the chopping branches dis contact with the cutter roller, it requires meeting the following in equation:

$$
\begin{gathered}
R_{A}-R>v t \quad(\mathrm{t}>0) \\
R_{A}-\sqrt{r^{2}+\left[\frac{\pi r(\theta-\omega t)}{180}\right]^{2}}>v t \quad(\mathrm{t}>0)
\end{gathered}
$$

The cutter roller rotate speed $\omega$ and feeding speed $v$ can be calculated according to the above formulas.

\section{Conclusion}

This paper discussed a new cutter roller which can be widely used in various branches chopping machine. This new cutter roller can solve the shortcomings of traditional chopping device, improve the efficiency of cutting machine, reduce the noise and prolong the service life of the cutter. And this kind of involute cutter roller can also be applied to other cutting device, and to improve the efficiency of cutting.

\section{Acknowledgements}

This paper is finacially supported by the Youth Science Fund of Nanchang Institute of Technology(Grant No. 2014KJ012)And Nanchang science and technology support project (Grant No. 2014-CXYHZ-ZBZZ-001)

\section{References}

[1]Lu Quanguo,Zhu Zhifang,Xia Shilong,Shuang Chaojun,Duan Niansong,Cha Ya. Design of New Mobile Branches Chopper for Biomass Power Generation [J]. Machine Design and Research ,2015,04:161-164.

[2]Zhao Jun. Mobile branch chopper control system design [D]. Chinese Academy of Agricultural mechanization sciences,2013.

[3]Zhu Dianxiang. Biomass pellet fuel production line and key manufacturing technology research and development [J]. Chinese Engineering Science,2014,04:13-16+44.

[4]Wang Cheng. Involute spur gear transmission system nonlinear dynamics research [D]. Beijing institute of technology,2015.

[5]Sun Jianguo. Involute cylindrical gear modification and the dynamic contact characteristics research [D]. Chongqing University,2008. 